\title{
Lie-Algebraic Approach for Pricing Zero-Coupon Bonds in Single-Factor Interest Rate Models
}

\author{
C. F. Lo \\ Institute of Theoretical Physics and Department of Physics, The Chinese University of Hong Kong, Shatin, New Territories, Hong Kong \\ Correspondence should be addressed to C. F. Lo; cflo@phy.cuhk.edu.hk
}

Received 17 December 2012; Revised 9 April 2013; Accepted 11 April 2013

Academic Editor: Alvaro Valencia

Copyright (c) 2013 C. F. Lo. This is an open access article distributed under the Creative Commons Attribution License, which permits unrestricted use, distribution, and reproduction in any medium, provided the original work is properly cited.

\begin{abstract}
The Lie-algebraic approach has been applied to solve the bond pricing problem in single-factor interest rate models. Four of the popular single-factor models, namely, the Vasicek model, Cox-Ingersoll-Ross model, double square-root model, and Ahn-Gao model, are investigated. By exploiting the dynamical symmetry of their bond pricing equations, analytical closed-form pricing formulae can be derived in a straightfoward manner. Time-varying model parameters could also be incorporated into the derivation of the bond price formulae, and this has the added advantage of allowing yield curves to be fitted. Furthermore, the Lie-algebraic approach can be easily extended to formulate new analytically tractable single-factor interest rate models.
\end{abstract}

\section{Introduction}

In this paper we apply the Lie-algebraic method to tackle the bond pricing problem in single-factor interest rate models. In particular, we investigate the bond pricing equation of the form

$$
\begin{aligned}
H(t) & B(r, t) \\
& \equiv\left\{\frac{1}{2} \sigma(t)^{2} r^{\nu} \frac{\partial^{2}}{\partial r^{2}}+\mu(r, t) \frac{\partial}{\partial r}-r\right\} B(r, t)=\frac{\partial B(r, t)}{\partial t},
\end{aligned}
$$

where $v$ is a real parameter, $\mu(r, t)$ is a real function of both spot interest rate $r$ and time-to-maturity $t, \sigma(t)$ is the timevarying volatility, and $B(r, t)$ denotes the price of a zerocoupon bond of duration $T$ with a value of unity at maturity; that is, $B(r, 0)=1$. By exploiting the dynamical symmetry $S U(1,1) \oplus h(1)$ of the bond pricing equation, we derive analytically tractable single-factor interest rate models in a unified manner and obtain their closed-form bond pricing formulae. It is found that not only four of the popular singlefactor models, namely the Vasicek model [1], Cox-IngersollRoss model [2], double square-root model [3], and AhnGao model [4], can be derived in a straightforward manner, but also new analytically tractable models can be formulated systematically. Moreover, time-varying model parameters can be incorporated into the derivation of the bond pricing formulae without difficulty. This has the added advantage of allowing yield curves to be fitted, and thus a "no-arbitrage" yield curve model can be developed to match the current market data.

The Lie-algebraic method was introduced by Lo and Hui [5-7] to the field of finance for the pricing of financial derivatives with time-dependent model parameters. This new method is very simple and consists of two basic ingredients: (1) identifying the dynamical symmetries of the given pricing partial differential equations and (2) applying the Wei-Norman theorem [8] to solve the equations and obtain analytical closed-form pricing formulae. For demonstration, the Lie-algebraic approach has already been applied to price European options for the constant elasticity of variance processes and corporate discount bonds with default risk, multiasset financial derivatives, and so forth. It should be noted that the Lie-algebraic method is different from the Lie group analysis which was introduced by Ibragimov and his coauthors $[9,10]$ to tackle partial differential equations occurring in financial problems. The Lie group analysis is a mathematical theory developed by Sophus Lie and classifies partial differential equations in terms of their symmetry groups, thereby identifying the set of equations which could 
be integrated or reduced to low-order equations by group theoretic algorithms. (Details of the Lie group analysis and its application to partial differential equations can be found in, for example, Hydon (2000) and Bluman et al. (2010) $[11,12]$.) Further applications of the Lie group analysis in mathematical finance were subsequently explored by a number of papers, for example, Goard [13], Pooe et al. [14], Goard et al. [15], Leach et al. [16], and Sinkala et al. [17]. A recent review of the applications of the Lie theory to problems in mathematical finance and economics can be found in [18].

\section{Lie-Algebraic Approach}

We consider a possible set of differential operators realizing the Lie algebra $S U(1,1) \oplus h(1)[19]$ :

$$
\begin{gathered}
W_{1}=r^{\gamma} \frac{\partial}{\partial r}-\lambda r^{\alpha}, \quad W_{2}=\frac{1}{1-\gamma} r^{1-\gamma}, \quad W_{3}=1 \\
K_{-} \equiv \frac{1}{2} W_{1}^{2}=\frac{1}{2} r^{2 \gamma} \frac{\partial^{2}}{\partial r^{2}}+\left(\frac{1}{2} \gamma r^{2 \gamma-1}-\lambda r^{\alpha+\gamma}\right) \frac{\partial}{\partial r} \\
+\frac{1}{2} \lambda r^{\alpha}\left(\lambda r^{\alpha}-\alpha r^{\gamma-1}\right), \\
K_{0} \equiv \frac{1}{4}\left(W_{1} W_{2}+W_{2} W_{1}\right)=\frac{1}{2(1-\gamma)} r \frac{\partial}{\partial r} \\
+\frac{1}{4}-\frac{\lambda}{2(1-\gamma)} r^{\alpha+1-\gamma}, \\
K_{+} \equiv \frac{1}{2} W_{2}^{2}=\frac{1}{2(1-\gamma)^{2}} r^{2(1-\gamma)},
\end{gathered}
$$

where $\alpha, \gamma$, and $\lambda$ are real adjustable parameters (see Appendix A). Then we try to look for appropriate linear combinations of these operators, namely, $H(t) \equiv A_{-}(t) K_{-}+$ $A_{0}(t) K_{0}+A_{+}(t) K_{+}+A_{1}(t) W_{1}+A_{2}(t) W_{2}+A_{3}(t) W_{3}$, where the coefficients are arbitrary scalar functions of $t$ only, to produce the bond pricing equation given in (1). Here are some illustrative examples.

(1) For $A_{-}(t)=\sigma(t)^{2}, A_{0}(t)=-4 A_{3}(t)=-2 \kappa(t)$, $A_{+}(t)=0, A_{1}(t)=\kappa(t) \theta(t), A_{2}(t)=-1, \gamma=\lambda=0$, and $\alpha$ being arbitrary, we recover the bond pricing equation of the Vasicek model:

$$
\left\{\frac{1}{2} \sigma(t)^{2} \frac{\partial^{2}}{\partial r^{2}}+\kappa(t)[\theta(t)-r] \frac{\partial}{\partial r}-r\right\} B(r, t)=\frac{\partial B(r, t)}{\partial t}
$$

By applying the Wei-Norman theorem, we can derive the bond price $B(r, t)$ as (see Appendix B)

$$
B(r, t)=U_{0}(t) U_{I}(t) B(r, 0), \quad B(r, 0)=1,
$$

where

$$
\begin{gathered}
U_{0}(t)=\exp \left\{c_{1}(t) K_{+}\right\} \exp \left\{c_{2}(t) K_{0}\right\} \exp \left\{c_{3}(t) K_{-}\right\}, \\
U_{I}(t)=\exp \left\{g_{1}(t) W_{1}\right\} \exp \left\{g_{2}(t) W_{2}\right\} \exp \left\{g_{3}(t) W_{3}\right\}, \\
c_{1}(t)=0, \\
c_{2}(t)=-2 \int_{0}^{t} \kappa(\tau) d \tau, \\
c_{3}(t)=\int_{0}^{t} \sigma(\tau)^{2} \exp \left\{c_{2}(\tau)\right\} d \tau, \\
g_{1}(t)=\int_{0}^{t}\left[\kappa(\tau) \theta(\tau) \exp \left\{\frac{1}{2} c_{2}(\tau)\right\}\right. \\
\left.+c_{3}(\tau) \exp \left\{-\frac{1}{2} c_{2}(\tau)\right\}\right] d \tau, \\
g_{2}(t)=-\int_{0}^{t} \exp \left\{-\frac{1}{2} c_{2}(\tau)\right\} d \tau, \\
g_{3}(t)=\int_{0}^{t}\left[\frac{1}{2} \kappa(\tau)+g_{1}(\tau) \exp \left\{-\frac{1}{2} c_{2}(\tau)\right\}\right] d \tau .
\end{gathered}
$$

As a result, the bond price $B(r, t)$ can be expressed as

$$
\begin{aligned}
B(r, t)= & \exp \left\{\frac{1}{4} c_{2}(t)+g_{3}(t)+g_{2}(t)\right. \\
\times & {\left.\left[g_{1}(t)+\frac{1}{2} c_{3}(t) g_{2}(t)\right]\right\} } \\
\times & \exp \left\{g_{2}(t) \exp \left[\frac{1}{2} c_{2}(t)\right] r\right\} .
\end{aligned}
$$

In the special case of constant model parameters, that is, $\sigma(t)=\sigma_{0}, \kappa(t)=\kappa_{0}$, and $\theta(t)=\theta_{0}$, the $c_{i}(t)$ and $g_{i}(t)$ can be analytically determined as

$$
\begin{gathered}
c_{1}(t)=0, \\
c_{2}(t)=-2 \kappa_{0} t, \\
c_{3}(t)=\frac{\sigma_{0}^{2}}{2}\left\{\frac{1-\exp \left(-2 \kappa_{0} t\right)}{\kappa_{0}}\right\}, \\
g_{1}(t)=\left(\kappa_{0} \theta_{0}-\frac{\sigma_{0}^{2}}{2 \kappa_{0}}\right)\left\{\frac{1-\exp \left(-2 \kappa_{0} t\right)}{\kappa_{0}}\right\} \\
+\frac{\sigma_{0}^{2}}{2 \kappa_{0}}\left\{\frac{\exp \left(\kappa_{0} t\right)-1}{\kappa_{0}}\right\}, \\
g_{2}(t)=-\left\{\frac{\exp \left(\kappa_{0} t\right)-1}{\kappa_{0}}\right\}, \\
\times\left\{\frac{1}{2} \kappa_{0} t-\left(\theta_{0}-\frac{\sigma_{0}^{2}}{2 \kappa_{0}^{2}}\right) t+\left(\theta_{0}-\frac{\sigma_{0}^{2}}{2 \kappa_{0}^{2}}\right)\right. \\
g_{3}\left(\kappa_{0} t\right)-1 \\
\left.\kappa_{0}\right\}+\frac{\sigma_{0}^{2}}{4 \kappa_{0}}\left\{\frac{\exp \left(\kappa_{0} t\right)-1}{\kappa_{0}}\right\}^{2},
\end{gathered}
$$


and the bond price $B(r, t)$ is reduced to the well-known closed-form expression [1]:

$$
\begin{aligned}
B(r, t)=\exp \{ & \left(\theta_{0}-\frac{\sigma_{0}^{2}}{2 \kappa_{0}^{2}}\right)\left[\frac{1-\exp \left(-\kappa_{0} t\right)}{\kappa_{0}}-t\right] \\
& -\frac{\sigma_{0}^{2}}{4 \kappa_{0}}\left[\frac{1-\exp \left(-\kappa_{0} t\right)}{\kappa_{0}}\right]^{2} \\
& \left.-\left[\frac{1-\exp \left(-\kappa_{0} t\right)}{\kappa_{0}}-t\right] r\right\} .
\end{aligned}
$$

(2) For $A_{-}(t)=\sigma(t)^{2}, A_{0}(t)=-4 A_{3}(t)=-2 \widetilde{\lambda}(t)$, $A_{+}(t)=-1 / 2, A_{1}(t)=-\kappa(t), A_{2}(t)=0, \gamma=1 / 2, \lambda=0$, and $\alpha$ being arbitrary, the bond pricing equation of the double square-root model is reproduced:

$$
\begin{gathered}
\left\{\frac{1}{2} \sigma(t)^{2} r \frac{\partial^{2}}{\partial r^{2}}+\left[\frac{1}{4} \sigma(t)^{2}-\kappa(t) \sqrt{r}-2 \widetilde{\lambda}(t) r\right] \frac{\partial}{\partial r}-r\right\} \\
\times B(r, t)=\frac{\partial B(r, t)}{\partial t} .
\end{gathered}
$$

As in the Vasicek model, we apply the Wei-Norman theorem to determine the bond price $B(r, t)$ as (see Appendix B)

$$
B(r, t)=U_{0}(t) U_{I}(t) B(r, 0), \quad B(r, 0)=1,
$$

where

$$
\begin{gathered}
U_{0}(t)=\exp \left\{c_{1}(t) K_{+}\right\} \exp \left\{c_{2}(t) K_{0}\right\} \exp \left\{c_{3}(t) K_{-}\right\}, \\
U_{I}(t)=\exp \left\{g_{1}(t) W_{1}\right\} \exp \left\{g_{2}(t) W_{2}\right\} \exp \left\{g_{3}(t) W_{3}\right\}, \\
\frac{d c_{1}(t)}{d t}=-1-2 \tilde{\lambda}(t) c_{1}(t)+\frac{1}{2} \sigma(t)^{2} c_{1}(t)^{2}, \quad c_{1}(0)=0, \\
c_{2}(t)=\int_{0}^{t}\left\{-2 \tilde{\lambda}(\tau)+\sigma(\tau)^{2} c_{1}(\tau)\right\} d \tau, \\
c_{3}(t)=\frac{1}{2} \int_{0}^{t} \sigma(\tau)^{2} \exp \left\{c_{2}(\tau)\right\} d \tau, \\
g_{1}(t)=\frac{1}{\sqrt{2}} \int_{0}^{t} \kappa(\tau)\left[c_{1}(\tau) c_{3}(\tau) \exp \left\{-\frac{1}{2} c_{2}(\tau)\right\}\right. \\
\left.g_{2}(t)=-\frac{1}{\sqrt{2}} \int_{0}^{t} \kappa(\tau) c_{1}(\tau) \exp \left\{-\frac{1}{2} c_{2}(\tau)\right\}\right] d \tau,
\end{gathered}
$$

$g_{3}(t)$

$$
=\int_{0}^{t}\left[\frac{1}{2} \tilde{\lambda}(\tau)+\frac{1}{\sqrt{2}} \kappa(\tau) c_{1}(\tau) \exp \left\{-\frac{1}{2} c_{2}(\tau)\right\} g_{1}(\tau)\right] d \tau .
$$

Accordingly, the bond price $B(r, t)$ is given by

$$
\begin{aligned}
B(r, t) & \\
= & \exp \left\{g_{3}(t)+g_{1}(t) g_{2}(t)\right. \\
& \left.+\frac{1}{2} c_{3}(t) g_{2}(t)^{2}+\frac{1}{4} c_{2}(t)\right\} \\
& \times \exp \left\{g_{2}(t) \exp \left[\frac{1}{2} c_{2}(t)\right] \sqrt{2 r}+c_{1}(t) r\right\} .
\end{aligned}
$$

In the special case of constant model parameters, that is, $\sigma(t)=\sigma_{0}, \kappa(t)=\kappa_{0}$, and $\tilde{\lambda}(t)=\widetilde{\lambda}_{0}$, the $c_{i}(t)$ and $g_{i}(t)$ can be analytically determined as

$$
\begin{aligned}
& c_{1}(t)=-\frac{2(\exp \{\gamma t\}-1)}{\left(\gamma+2 \widetilde{\lambda}_{0}\right)(\exp \{\gamma t\}-1)+2 \gamma} \\
& =\frac{2 \tilde{\lambda}_{0}-\gamma}{\sigma_{0}^{2}}+\frac{2 \gamma}{\sigma_{0}^{2}\left[1-C_{0} \exp \{\gamma t\}\right]}, \\
& c_{2}(t)=-2 \tilde{\lambda}_{0} t \\
& +2 \ln \left\{\frac{2 \gamma \exp \left[(1 / 2)\left(\gamma+2 \tilde{\lambda}_{0}\right) t\right]}{\left(\gamma+2 \widetilde{\lambda}_{0}\right)(\exp \{\gamma t\}-1)+2 \gamma}\right\} \\
& =\gamma t+2 \ln \left\{\frac{2 \gamma}{\left(\gamma-2 \widetilde{\lambda}_{0}\right)\left[1-C_{0} \exp \{\gamma t\}\right]}\right\}, \\
& c_{3}(t)=-\frac{1}{2} \sigma_{0}^{2} c_{1}(t), \\
& g_{1}(t)=\frac{\sqrt{2} \kappa_{0} \widetilde{\lambda}_{0}}{\gamma^{2}} \exp \left\{-\frac{1}{2} \gamma t\right\}\left(1-\exp \left\{\frac{1}{2} \gamma t\right\}\right)^{2} \\
& -\frac{\kappa_{0}}{\sqrt{2} \gamma}\left(\exp \left\{\frac{1}{2} \gamma t\right\}-\exp \left\{-\frac{1}{2} \gamma t\right\}\right) \text {, } \\
& g_{2}(t)=\frac{\sqrt{2} \kappa_{0}}{\gamma^{2}} \exp \left\{-\frac{1}{2} \gamma t\right\}\left(1-\exp \left\{\frac{1}{2} \gamma t\right\}\right)^{2} \text {, } \\
& g_{3}(t)=\left(\frac{1}{2} \widetilde{\lambda}_{0}-\frac{\kappa_{0}^{2}}{\gamma^{2}}\right) t-\frac{\kappa_{0}^{2} \widetilde{\lambda}_{0}}{\gamma^{4}} \exp \{-\gamma t\} \\
& \times\left(1-\exp \left\{\frac{1}{2} \gamma t\right\}\right)^{4} \\
& +\frac{\kappa_{0}^{2}}{2 \gamma^{3}}(\exp \{\gamma t\}-\exp \{-\gamma t\})
\end{aligned}
$$

with $\gamma=\sqrt{4 \tilde{\lambda}_{0}^{2}+2 \sigma_{0}^{2}}$ and $C_{0}=\left(2 \tilde{\lambda}_{0}+\gamma\right) /\left(2 \tilde{\lambda}_{0}-\gamma\right)$. Consequently, we are able to recover the well-known closedform expression of the bond price $B(r, t)[3]$ :

$$
B(r, t)=\Psi(t) \exp \{\Omega(t) r+\Gamma(t) \sqrt{r}\},
$$


where

$$
\begin{gathered}
\Psi(t)=\sqrt{\frac{1-C_{0}}{1-C_{0} \exp \{\gamma t\}}} \\
\quad \times \exp \left(\alpha_{1}+\alpha_{2} t+\frac{\alpha_{3}+\alpha_{4} \exp \{(1 / 2) \gamma t\}}{1-C_{0} \exp \{\gamma t\}}\right), \\
\Omega(t)=\frac{2 \tilde{\lambda}_{0}-\gamma}{\sigma_{0}^{2}}+\frac{2 \gamma}{\sigma_{0}^{2}\left[1-C_{0} \exp \{\gamma t\}\right]}, \\
\Gamma(t)=\frac{2 \kappa_{0}\left(2 \tilde{\lambda}_{0}+\gamma\right)(1-\exp \{(1 / 2) \gamma t\})^{2}}{\gamma \sigma_{0}^{2}\left[1-C_{0} \exp \{\gamma t\}\right]},
\end{gathered}
$$

with

$$
\begin{gathered}
\alpha_{1}=-\frac{\kappa_{0}^{2}}{\gamma^{3} \sigma_{0}^{2}}\left(4 \tilde{\lambda}_{0}+\gamma\right)\left(2 \tilde{\lambda}_{0}-\gamma\right), \\
\alpha_{2}=\frac{2 \tilde{\lambda}_{0}+\gamma}{4}-\frac{\kappa_{0}^{2}}{\gamma^{2}}, \\
\alpha_{3}=\frac{4 \kappa_{0}^{2}}{\gamma^{3} \sigma_{0}^{2}}\left(2 \tilde{\lambda}_{0}^{2}-\sigma_{0}^{2}\right), \\
\alpha_{4}=-\frac{8 \kappa_{0}^{2} \tilde{\lambda}_{0}}{\gamma^{3} \sigma_{0}^{2}}\left(2 \tilde{\lambda}_{0}+\gamma\right) .
\end{gathered}
$$

(3) For $A_{-}(t)=\sigma(t)^{2}, A_{0}(t)=-4 A_{3}(t) / 3, A_{+}(t)=$ $A_{1}(t)=0, A_{2}(t)=-1, \gamma=0$, and $\lambda=\alpha=-1$, a bond pricing equation with a special time-dependent nonlinear drift term can be obtained:

$$
\begin{gathered}
\left\{\frac{1}{2} \sigma(t)^{2} \frac{\partial^{2}}{\partial r^{2}}+\left[\frac{1}{2} A_{0}(t) r+\frac{\sigma(t)^{2}}{r}\right] \frac{\partial}{\partial r}-r\right\} B(r, t) \\
=\frac{\partial B(r, t)}{\partial t}
\end{gathered}
$$

which can be straightforwardly solved as in the Vasicek model. As a result, the bond price $B(r, t)$ can be expressed as (see Appendix B)

$$
\begin{aligned}
B(r, t)= & \exp \left\{\frac{3}{4} c_{2}(t)+g_{3}(t)+g_{2}(t)\right. \\
& \left.\times\left(g_{1}(t)+\frac{1}{2} c_{3}(t) g_{2}(t)\right)\right\} \\
\times & \exp \left\{g_{2}(t) \exp \left(\frac{1}{2} c_{2}(t)\right) r\right\} \\
\times & \times\left\{1+\left[g_{1}(t)+c_{3}(t) g_{2}(t)\right] \exp \left(\frac{1}{2} c_{2}(t)\right) \frac{1}{r}\right\},
\end{aligned}
$$

where

$$
\begin{gathered}
c_{2}(t)=\int_{0}^{t} A_{0}(\tau) d \tau \\
c_{3}(t)=\int_{0}^{t} \sigma(\tau)^{2} \exp \left\{c_{2}(\tau)\right\} d \tau \\
g_{1}(t)=\int_{0}^{t} c_{3}(\tau) \exp \left\{-\frac{1}{2} c_{2}(\tau)\right\} d \tau \\
g_{2}(t)=-\int_{0}^{t} \exp \left\{-\frac{1}{2} c_{2}(\tau)\right\} d \tau \\
g_{3}(t)=\int_{0}^{t}\left[-\frac{3}{4} A_{0}(\tau)+g_{1}(\tau) \exp \left\{-\frac{1}{2} c_{2}(\tau)\right\}\right] d \tau .
\end{gathered}
$$

(4) For $A_{-}(t)=\sigma(t)^{2}, A_{0}(t)=-4 A_{3}(t) / 3, A_{+}(t)=$ $A_{2}(t)=0, A_{1}(t)=1, \gamma=2$, and $\lambda=\alpha=1$, we can derive the bond pricing equation:

$$
\begin{gathered}
\left\{\frac{1}{2} \sigma(t)^{2} r^{4} \frac{\partial^{2}}{\partial r^{2}}+\left[r^{2}-\frac{1}{2} A_{0}(t) r\right] \frac{\partial}{\partial r}-r\right\} B(r, t) \\
=\frac{\partial B(r, t)}{\partial t},
\end{gathered}
$$

which has both the $r^{2}$ dependence of volatility and a timedependent nonlinear drift term. By performing the same analysis as in the other three cases, the bond price $B(r, t)$ is found to be given by (see Appendix B)

$$
B(r, t)=1-g_{1}(t) \exp \left\{-\frac{1}{2} c_{2}(t)\right\} r,
$$

where

$$
\begin{gathered}
c_{2}(t)=\int_{0}^{t} A_{0}(\tau) d \tau, \\
g_{1}(t)=\int_{0}^{t} \exp \left\{\frac{1}{2} c_{2}(\tau)\right\} d \tau .
\end{gathered}
$$

Next we apply the same analysis to derive the CoxIngersoll-Ross model and Ahn-Gao model from an alternative set of differential operators realizing the subalgebra $\mathfrak{Q}$ of the Lie algebra $S U(1,1) \oplus h(1)$ :

$$
\begin{gathered}
K_{-}=\frac{1}{2} r^{2 \gamma} \frac{\partial^{2}}{\partial r^{2}}+\left(\frac{1}{2} \gamma-\lambda\right) r^{2 \gamma-1} \frac{\partial}{\partial r}+\alpha r^{2 \gamma-2}, \\
K_{0}=\frac{1}{2(1-\gamma)} r \frac{\partial}{\partial r}+\frac{1}{4}-\frac{\lambda}{2(1-\gamma)}, \\
K_{+}=\frac{1}{2(1-\gamma)^{2}} r^{2(1-\gamma)} \\
W_{3}=1,
\end{gathered}
$$

where $\alpha, \gamma$, and $\lambda$ are real adjustable parameters (see Appendix A). The subalgebra $\mathbb{Q}$ is actually the reductive Lie algebra $U(1,1)$. 
(1) By choosing $\alpha=0, \gamma=1 / 2$, and $\lambda=1 / 4-\kappa \theta / \sigma^{2}$, the bond pricing equation of the Cox-Ingersoll-Ross model with constant model parameters can be expressed in terms of the differential operators realizing the subalgebra $\mathbb{Q}$ as

$$
\begin{aligned}
\frac{\partial B(r, t)}{\partial t} & =\left\{\frac{1}{2} \sigma^{2} r \frac{\partial^{2}}{\partial r^{2}}+\kappa(\theta-r) \frac{\partial}{\partial r}-r\right\} B(r, t) \\
& =\left\{\sigma^{2} K_{-}-\kappa K_{0}-\frac{1}{2} K_{+}+\frac{\kappa^{2} \theta}{\sigma^{2}} W_{3}\right\} B(r, t) .
\end{aligned}
$$

(Strictly speaking, the model parameters $\kappa, \theta$, and $\sigma$ could be time-varying with the constraint that $\kappa \theta / \sigma^{2}$ is independent of time t.)

By the Wei-Norman theorem, the bond price $B(r, t)$ can be easily determined as (see Appendix B)

$$
B(r, t)=\exp \left\{2 c_{1}(t) r\right\} \exp \left\{\frac{\kappa \theta}{\sigma^{2}}\left[\kappa t+c_{2}(t)\right]\right\},
$$

where

$$
\begin{gathered}
\frac{d c_{1}(t)}{d t}=-\frac{1}{2}-\kappa c_{1}(t)+\sigma^{2} c_{1}(t)^{2}, \quad c_{1}(0)=0, \\
c_{2}(t)=-\kappa t+\sigma^{2} \int_{0}^{t} c_{1}(\tau) d \tau .
\end{gathered}
$$

The Riccati equation with constant coefficients in (26) can be straightforwardly solved to yield

$$
c_{1}(t)=-\frac{\exp \{\gamma t\}-1}{(\gamma+\kappa)(\exp \{\gamma t\}-1)+2 \gamma}
$$

with $\gamma=\sqrt{\kappa^{2}+2 \sigma^{2}}$. Once the $c_{1}(t)$ has been found, we are also able to obtain

$$
c_{2}(t)=-\kappa t+2 \ln \left(\frac{2 \gamma \exp \{(1 / 2)(\gamma+\kappa) t\}}{(\gamma+\kappa)(\exp \{\gamma t\}-1)+2 \gamma}\right)
$$

via analytical integrations. Beyond question, our finding is in agreement with the well-known closed-form result [2]:

$$
\begin{aligned}
B(r, t)= & \left(\frac{2 \gamma \exp \{(1 / 2)(\gamma+\kappa) t\}}{(\gamma+\kappa)(\exp \{\gamma t\}-1)+2 \gamma}\right)^{2 \kappa \theta / \sigma^{2}} \\
& \times \exp \left\{-\frac{2(\exp \{\gamma t\}-1) r}{(\gamma+\kappa)(\exp \{\gamma t\}-1)+2 \gamma}\right\} .
\end{aligned}
$$

(2) By setting $\alpha=-1 / \sigma^{2}, \gamma=3 / 2$, and $\lambda=q+3 / 4$, we can cast the bond pricing equation of the Ahn-Gao model, which includes nonlinearity in the drift term and a realistic $r^{3 / 2}$ dependence in the volatility (not only the nonlinear drift term of the Ahn-Gao model is consistent with the empirical findings of Aït-Sahalia [20], but also the chosen $r^{3 / 2}$ dependence of volatility is the best fit power law for volatility $[21,22])$, in terms of the differential operators realizing the subalgebra $\mathfrak{Q}$ into the form

$$
\begin{aligned}
\frac{\partial B(r, t)}{\partial t} & =\left\{\frac{1}{2} \sigma^{2} r^{3} \frac{\partial^{2}}{\partial r^{2}}+\sigma^{2}\left[a(t) r-q r^{2}\right] \frac{\partial}{\partial r}-r\right\} B(r, t) \\
& =\left\{\sigma^{2} K_{-}-\sigma^{2} a(t) K_{0}+\sigma^{2}(q+1) a(t) W_{3}\right\} B(r, t),
\end{aligned}
$$

where $a(t)$ is a real function of $t$. Then applying the WeiNorman theorem allows us to represent the bond price $B(r, t)$ by (see Appendix B)

$$
\begin{aligned}
B(r, t)= & \exp \left\{\sigma^{2}(q+1) \int_{0}^{t} a(\tau) d \tau\right\} \exp \left\{c_{2}(t) K_{0}\right\} \\
& \times \exp \left\{c_{3}(t) K_{-}\right\} B(r, 0),
\end{aligned}
$$

where $B(r, 0)=1$ and

$$
\begin{gathered}
c_{2}(t)=-\sigma^{2} \int_{0}^{t} a(\tau) d \tau, \\
c_{3}(t)=\sigma^{2} \int_{0}^{t} \exp \left\{c_{2}(\tau)\right\} d \tau .
\end{gathered}
$$

Without loss of generality, we suppose that $B(r, 0)=$ $x^{-(2 q+1)} V(x)$, where $x=2 / \sqrt{r}$,

$$
V(x)=\int_{0}^{\infty} d v v J_{p}(x v) \int_{0}^{\infty} d y y J_{p}(y v) V(y),
$$

and $p=\sqrt{(2 q+1)^{2}+8 / \sigma^{2}}$. It is not difficult to show that the bond price $B(r, t)$ is given by

$$
B(r, t)=\int_{0}^{\infty} d x^{\prime} G\left(x, t ; x^{\prime}, 0\right) B\left(r^{\prime}, 0\right),
$$

where $x^{\prime}=2 / \sqrt{r^{\prime}}$ and

$$
\begin{aligned}
G\left(x, t ; x^{\prime}, 0\right)= & x^{\prime}\left[\frac{x^{\prime}}{x \exp \left\{c_{2}(t) / 2\right\}}\right]^{2 q+1} \\
& \times \int_{0}^{\infty} d v v J_{p}\left(x v \exp \left\{\frac{c_{2}(t)}{2}\right\}\right) \\
& \times J_{p}\left(x^{\prime} v\right) \exp \left\{-\frac{c_{3}(t)}{2} v^{2}\right\} .
\end{aligned}
$$

The function $J_{p}(\xi)$ is the Bessel function of the first kind of order $p$. Here we have made use of the fact that $x^{-(2 q+1)} J_{p}(x v)$ is an eigenfunction of the operator $K_{-}$with the eigenvalue $-v^{2} / 2$. The integral over $v$ can be analytically evaluated to give [23]

$$
\frac{1}{c_{3}(t)} \exp \left\{-\frac{x^{\prime 2}+x^{2} \exp \left\{c_{2}(t)\right\}}{2 c_{3}(t)}\right\} I_{p}\left(\frac{x^{\prime} x \exp \left\{c_{2}(t) / 2\right\}}{c_{3}(t)}\right)
$$

for $p>-1, x^{\prime}>0, x \exp \left\{c_{2}(t) / 2\right\}>0$, and $\left|\arg \left[c_{2}(t) / 2\right]^{1 / 2}\right|<$ $\pi / 4$. The function $I_{p}(\xi)$ is the modified Bessel function of the first kind of order $p$. As a result, $G\left(x, t ; x^{\prime}, 0\right)$ is found to be given by

$$
\begin{aligned}
G\left(x, t ; x^{\prime}, 0\right)= & \frac{x^{\prime}}{c_{3}(t) \exp \left\{(2 q+1) c_{2}(t) / 2\right\}}\left(\frac{x^{\prime}}{x}\right)^{2 q+1} \\
& \times I_{p}\left(\frac{x^{\prime} x \exp \left\{c_{2}(t) / 2\right\}}{c_{3}(t)}\right) \\
& \times \exp \left\{-\frac{x^{\prime 2}+x^{2} \exp \left\{c_{2}(t)\right\}}{2 c_{3}(t)}\right\} .
\end{aligned}
$$


Since $B(r, 0)=1$, we can readily derive the bond price $B(r, t)$ as follows:

$$
\begin{aligned}
B(r, t)= & \frac{x^{-(2 q+1)}}{c_{3}(t) \exp \left\{(2 q+1) c_{2}(t) / 2\right\}} \\
& \times \exp \left\{-\frac{x^{2} \exp \left\{c_{2}(t)\right\}}{2 c_{3}(t)}\right\} \\
& \times \int_{0}^{\infty} d x^{\prime} x^{\prime 2(q+1)} \exp \left\{-\frac{x^{\prime 2}}{2 c_{3}(t)}\right\} \\
& \times I_{p}\left(\frac{x^{\prime} x \exp \left\{c_{2}(t) / 2\right\}}{c_{3}(t)}\right) \\
= & \frac{\Gamma(p+1-\omega)}{\Gamma(p+1)} M\left(\omega, p+1,-\frac{2 \exp \left\{c_{2}(t)\right\}}{c_{3}(t) r}\right) \\
& \times\left\{\frac{2 \exp \left\{c_{2}(t)\right\}}{c_{3}(t) r}\right\},
\end{aligned}
$$

where $\omega=-(2 q+1-p) / 2, \Gamma(\xi)$ denotes the Gamma function and $M(\xi, \chi, \rho)$ is the standard confluent hypergeometric function $[23,24]$. Furthermore, (38) will reproduce the wellknown closed-form result if the model parameter $a(t)$ is independent of time [4].

\section{Conclusion}

In this paper the Lie-algebraic method has been applied to solve the bond pricing problem in single-factor interest rate models. Four of the popular single-factor models, namely the Vasicek model, Cox-Ingersoll-Ross model, double squareroot model, and Ahn-Gao model, are investigated, and analytical closed-form pricing formulae are derived. Since all the four bond pricing equations exhibit the dynamical symmetry $S U(1,1) \oplus h(1)$ or its subgroup, their solutions can be derived in a unified manner and have very similar mathematical structures. This interesting feature helps shed new light upon the systematic formulation of new analytically tractable single-factor interest rate models, as demonstrated in Section 2. Time-varying model parameters could also be incorporated into the derivation of the bond price formulae without difficulty. This has the added advantage of allowing yield curves to be fitted, and thus a "no-arbitrage" yield curve model can be developed to match the current market data. Hence, we believe that the Lie-algebraic method will provide an easy-to-use analytical tool for the bond pricing problem. Furthermore, the Lie-algebraic approach can be easily extended to the pricing of other standard European interest rate derivatives for they differ from the zero-coupon bonds in the final payoff conditions only [25].

\section{Appendices}

A. Generators of the Lie Algebra $S U(1,1) \oplus h(1)$
and Its Subalgebras

The generators $\left\{W_{1}, W_{2}, W_{3}\right\}$ of the Heisenberg-Weyl Lie algebra $h(1)$ obey the set of commutation relations [19]:

$$
\left[W_{1}, W_{2}\right]=W_{3}, \quad\left[W_{1}, W_{3}\right]=\left[W_{2}, W_{3}\right]=0 .
$$

By direct substitution, a possible set of differential operators realizing the Lie algebra can be identified as

$$
\begin{gathered}
W_{1}=r^{\gamma} \frac{\partial}{\partial r}-\lambda r^{\alpha}, \\
W_{2}=\frac{1}{1-\gamma} r^{1-\gamma}, \\
W_{3}=1,
\end{gathered}
$$

where $\alpha, \gamma$, and $\lambda$ are real adjustable parameters. Then, in terms of these generators one can construct the generators $\left\{K_{+}, K_{0}, K_{-}\right\}$of the Lie algebra $S U(1,1)$ as follows:

$$
\begin{aligned}
& K_{-} \equiv \frac{1}{2} W_{1}^{2} \\
&= \frac{1}{2} r^{2 \gamma} \frac{\partial^{2}}{\partial r^{2}}+\left(\frac{1}{2} \gamma r^{2 \gamma-1}-\lambda r^{\alpha+\gamma}\right) \frac{\partial}{\partial r} \\
&+\frac{1}{2} \lambda r^{\alpha}\left(\lambda r^{\alpha}-\alpha r^{\gamma-1}\right), \\
& K_{0} \equiv \frac{1}{4}\left(W_{1} W_{2}+W_{2} W_{1}\right) \\
&= \frac{1}{2(1-\gamma)} r \frac{\partial}{\partial r}+\frac{1}{4}-\frac{\lambda}{2(1-\gamma)} r^{\alpha+1-\gamma}, \\
& K_{+} \equiv \frac{1}{2} W_{2}^{2}=\frac{1}{2(1-\gamma)^{2}} r^{2(1-\gamma)}
\end{aligned}
$$

which satisfy the set of commutation relations [19]:

$$
\left[K_{+}, K_{-}\right]=-2 K_{0}, \quad\left[K_{0}, K_{ \pm}\right]= \pm K_{ \pm} \text {. }
$$

These six generators $\left\{W_{1}, W_{2}, W_{3}, K_{+}, K_{0}, K_{-}\right\}$in turn form the Lie algebra $S U(1,1) \oplus h(1)$, which is defined by the following set of commutation relations [19]:

$$
\begin{gathered}
{\left[W_{1}, W_{2}\right]=W_{3}, \quad\left[W_{1}, W_{3}\right]=\left[W_{2}, W_{3}\right]=0,} \\
{\left[K_{+}, K_{-}\right]=-2 K_{0}, \quad\left[K_{0}, K_{ \pm}\right]= \pm K_{ \pm},} \\
{\left[W_{1}, K_{+}\right]=W_{2}, \quad\left[W_{1}, K_{0}\right]=\frac{1}{2} W_{1},} \\
{\left[W_{2}, K_{0}\right]=-\frac{1}{2} W_{2}, \quad\left[W_{2}, K_{-}\right]=-W_{1},} \\
{\left[W_{1}, K_{-}\right]=\left[W_{2}, K_{+}\right]=\left[W_{3}, K_{0}\right]=\left[W_{3}, K_{ \pm}\right]=0 .}
\end{gathered}
$$


In addition to the subalgebras $S U(1,1)$ and $h(1)$, we may also form another subalgebra $\mathbb{Q}$ in terms of the four generators $\left\{W_{3}, K_{+}, K_{0}, K_{-}\right\}$satisfying the commutation relations:

$$
\begin{gathered}
{\left[K_{+}, K_{-}\right]=-2 K_{0}, \quad\left[K_{0}, K_{ \pm}\right]= \pm K_{ \pm}} \\
{\left[W_{3}, K_{0}\right]=\left[W_{3}, K_{ \pm}\right]=0}
\end{gathered}
$$

The subalgebra $\mathbb{Q}$ is actually the reductive Lie algebra $U(1,1)$. Moreover, it is not difficult to show that an alternative realization of the set of generators of the Lie algebra $\mathbb{Q}$ is given by

$$
\begin{gathered}
K_{-}=\frac{1}{2} r^{2 \gamma} \frac{\partial^{2}}{\partial r^{2}}+\left(\frac{1}{2} \gamma-\lambda\right) r^{2 \gamma-1} \frac{\partial}{\partial r}+\alpha r^{2 \gamma-2}, \\
K_{0}=\frac{1}{2(1-\gamma)} r \frac{\partial}{\partial r}+\frac{1}{4}-\frac{\lambda}{2(1-\gamma)}, \\
K_{+}=\frac{1}{2(1-\gamma)^{2}} r^{2(1-\gamma)}, \\
W_{3}=1,
\end{gathered}
$$

where $\alpha, \gamma$, and $\lambda$ are real adjustable parameters.

\section{B. Wei-Norman Theorem}

Consider the linear operator differential equation of the first order

$$
\frac{d U(t)}{d t}=H(t) U(t), \quad U(0)=1,
$$

where $H$ and $U$ are both time-dependent linear operators in a Banach space or a finite-dimensional space. According to the Wei-Norman theorem [8], if the operator $H$ can be expressed as

$$
H(t)=\sum_{n=1}^{N} a_{n}(t) L_{n}
$$

where $a_{n}$ 's are scalar functions of time and $L_{n}$ are the generators of an $\mathrm{N}$-dimensional solvable Lie algebra or a real split 3-dimensional simple Lie algebra, then the operator $U$ can assume the following form:

$$
U(t)=\prod_{n=1}^{N} \exp \left\{g_{n}(t) L_{n}\right\} .
$$

Here the $g_{n}$ 's are time-dependent scalar functions to be determined. To find the $g_{n}$ 's, we simply substitute (B.2) and (B.3) into (B.1) and compare the two sides term by term to obtain a set of coupled nonlinear differential equations

$$
\frac{d g_{n}(t)}{d t}=\sum_{m=1}^{N} \eta_{n m} a_{m}(t), \quad g_{n}(0)=0
$$

where $\eta_{n m}$ are nonlinear functions of $g_{n}$ 's. Thus, we have transformed the linear operator differential equation in (B.1) to a set of coupled nonlinear differential equations of scalar functions in (B.4).

Moreover, according to Levi's Theorem, "If $L$ is a finitedimensional Lie algebra with radical $R$ which is the maximal solvable ideal of the Lie algebra, then there exists a semisimple subalgebra $S$ of $L$ such that $L$ is the semidirect sum $L=S \oplus R$," in the equation $d U(t) / d t=H(t) U(t)$, where $H(t)$ generates $L$, the decomposition $L=S \oplus R$ gives rise to the corresponding decomposition $H(t)=H_{S}(t)+H_{R}(t)$, where $H_{S}(t) \in S$ and $H_{R}(t) \in R$. Then it is easy to verify that $U(t)=U_{S}(t) U_{R}(t)$ where $U_{S}(t)$ and $U_{R}(t)$ satisfy

$$
\begin{gathered}
\frac{\partial U_{S}(t)}{\partial \tau}=H_{S}(t) U_{S}(t), \\
\frac{\partial U_{R}(t)}{\partial t}=\left\{U_{S}(t)^{-1} H_{R}(t) U_{S}(t)\right\} U_{R}(t) .
\end{gathered}
$$

Since $R$ is an ideal in $L$, we can easily see that $U_{S}(t)^{-1}$ $H_{R}(t) U_{S}(t)$ is in $R$. The fact that $R$ is solvable makes it easy to find $U_{R}(t)$ once $U_{S}(t)$ has been found. More details can be found in [8].

For illustration, we apply the Wei-Norman theorem to the following cases.

B.1. Heisenberg-Weyl Lie Algebra h(1). The Heisenberg-Weyl Lie algebra $h(1)$ is defined by the set of commutation relations [17]:

$$
\left[W_{1}, W_{2}\right]=W_{3}, \quad\left[W_{1}, W_{3}\right]=\left[W_{2}, W_{3}\right]=0,
$$

of its generators $\left\{W_{1}, W_{2}, W_{3}\right\}$. Given that

$$
H(t)=a_{1}(t) W_{1}+a_{2}(t) W_{2}+a_{3}(t) W_{3},
$$

the Wei-Norman theorem states that $U(t)$ can be expressed as

$$
U(t)=\exp \left\{g_{1}(t) W_{1}\right\} \exp \left\{g_{2}(t) W_{2}\right\} \exp \left\{g_{3}(t) W_{3}\right\},
$$

where the time-dependent functions $g_{n}$ 's satisfy a set of three coupled nonlinear differential equations:

$$
\begin{gathered}
\frac{d g_{1}(t)}{d t}=a_{1}(t), \\
\frac{d g_{2}(t)}{d t}=a_{2}(t), \\
\frac{d g_{3}(t)}{d t}+g_{1}(t) \frac{d g_{2}(t)}{d t}=a_{3}(t) .
\end{gathered}
$$

It is obvious that the set of differential equations can be easily solved by quadrature:

$$
\begin{gathered}
g_{1}(t)=\int_{0}^{t} d \tau a_{1}(\tau), \\
g_{2}(t)=\int_{0}^{t} d \tau a_{2}(\tau), \\
g_{3}(t)=\int_{0}^{t} d \tau\left[a_{3}(\tau)-a_{2}(\tau) g_{1}(\tau)\right] .
\end{gathered}
$$

As a result, the operator $U(t)$ is thus determined. 
B.2. SU $(1,1)$ Lie Algebra. We consider the evolution equation of the operator $U(t)$ :

$$
\begin{array}{r}
\frac{d U(t)}{d t}=\left\{a_{1}(t) K_{+}+a_{2}(t) K_{0}+a_{3}(t) K_{-}\right\} U(t), \\
U(0)=1,
\end{array}
$$

where the operators $\left\{K_{+}, K_{0}, K_{-}\right\}$form the $S U(1,1)$ Lie algebra defined by the commutation relations [19]:

$$
\left[K_{+}, K_{-}\right]=-2 K_{0}, \quad\left[K_{0}, K_{ \pm}\right]= \pm K_{ \pm} .
$$

According to the Wei-Norman theorem, the operator $U(t)$ can be expressed in the product form

$$
\begin{array}{r}
U(t)=\exp \left\{c_{1}(t) K_{+}\right\} \exp \left\{c_{2}(t) K_{0}\right\} \exp \left\{c_{3}(t) K_{-}\right\} \\
c_{i}(0)=0
\end{array}
$$

where the time-dependent functions $c_{n}$ 's satisfy a set of three coupled nonlinear differential equations:

$$
\begin{gathered}
\frac{d c_{1}(t)}{d t}-c_{1}(t) \frac{d c_{2}(t)}{d t}+c_{1}(t)^{2} \exp \left\{-c_{2}(t)\right\} \frac{d c_{3}(t)}{d t}=a_{1}(t), \\
\frac{d c_{2}(t)}{d t}-2 c_{1}(t) \exp \left\{-c_{2}(t)\right\} \frac{d c_{3}(t)}{d t}=a_{2}(t), \\
\exp \left\{-c_{2}(t)\right\} \frac{d c_{3}(t)}{d t}=a_{3}(t) .
\end{gathered}
$$

After further simplification, these three differential equations become

$$
\begin{gathered}
\frac{d c_{1}(t)}{d t}=a_{1}(t)+a_{2}(t) c_{1}(t)+a_{3}(t) c_{1}(t)^{2}, \quad c_{1}(0)=0 \\
c_{2}(t)=\int_{0}^{t}\left\{a_{2}(\tau)+2 a_{3}(\tau) c_{1}(\tau)\right\} d \tau \\
c_{3}(t)=\int_{0}^{t} a_{3}(\tau) \exp \left\{c_{2}(\tau)\right\} d \tau .
\end{gathered}
$$

Hence, once the $c_{1}(t)$ is found by solving the Riccati equation, the $c_{2}(t)$ and $c_{3}(t)$ can be readily determined by quadrature.

B.3. $S U(1,1) \oplus h(1)$ Lie Algebra. If $H(t)$ is a linear combination of the six generators $\left\{W_{1}, W_{2}, W_{3}, K_{+}, K_{0}, K_{-}\right\}$of the Lie algebra $S U(1,1) \oplus h(1)$, then, according to Levi's theorem, we may decompose the $H(t)$ into two parts $H_{S}(t)=a_{1}(t) K_{+}+$ $a_{2}(t) K_{0}+a_{3}(t) K_{-}$and $H_{R}(t)=b_{1}(t) W_{1}+b_{2}(t) W_{2}+b_{3}(t) W_{3}$, and the operator $U(t)$ assumes the product form $U(t)=$ $U_{S}(t) U_{R}(t)$ where $U_{S}(t)$ and $U_{R}(t)$ satisfy (B.5) and (B.6), respectively. It is obvious that the operator $U_{S}(t)$ is given by

$$
U_{S}(t)=\exp \left\{c_{1}(t) K_{+}\right\} \exp \left\{c_{2}(t) K_{0}\right\} \exp \left\{c_{3}(t) K_{-}\right\},
$$

where

$$
\begin{gathered}
\frac{d c_{1}(t)}{d t}=a_{1}(t)+a_{2}(t) c_{1}(t)+a_{3}(t) c_{1}(t)^{2}, \quad c_{1}(0)=0, \\
c_{2}(t)=\int_{0}^{t}\left\{a_{2}(\tau)+2 a_{3}(\tau) c_{1}(\tau)\right\} d \tau, \\
c_{3}(t)=\int_{0}^{t} a_{3}(\tau) \exp \left\{c_{2}(\tau)\right\} d \tau .
\end{gathered}
$$

Next, in order to determine $U_{R}(t)$, we need to evaluate $H_{I}(t) \equiv U_{S}(t)^{-1} H_{R}(t) U_{S}(t)$. Using the explicit form of the operator $U_{S}(t)$, we can apply the Baker-Hausdorff formula [26] to derive the operator $H_{I}(t)$ :

$$
H_{I}(t)=\bar{a}_{1}(t) W_{1}+\bar{a}_{2}(t) W_{2}+\bar{a}_{3}(t) W_{3},
$$

where

$$
\begin{gathered}
\bar{a}_{1}(t)=b_{1}(t) \exp \left\{\frac{1}{2} c_{2}(t)\right\}-\left[b_{1}(t) c_{1}(t)+b_{2}(t)\right] \\
\times c_{3}(t) \exp \left\{-\frac{1}{2} c_{2}(t)\right\}, \\
\bar{a}_{2}(t)=\left[b_{1}(t) c_{1}(t)+b_{2}(t)\right] \exp \left\{-\frac{1}{2} c_{2}(t)\right\}, \\
\bar{a}_{3}(t)=b_{3}(t) .
\end{gathered}
$$

Then, the operator $U_{R}(t)$ can be easily found to be given by

$$
U_{R}(t)=\exp \left\{g_{1}(t) W_{1}\right\} \exp \left\{g_{2}(t) W_{2}\right\} \exp \left\{g_{3}(t) W_{3}\right\},
$$

where

$$
\begin{gathered}
g_{1}(t)=\int_{0}^{t} d \tau \bar{a}_{1}(\tau), \\
g_{2}(t)=\int_{0}^{t} d \tau \bar{a}_{2}(\tau), \\
g_{3}(t)=\int_{0}^{t} d \tau\left[\bar{a}_{3}(\tau)-\bar{a}_{2}(\tau) g_{1}(\tau)\right] .
\end{gathered}
$$

\section{References}

[1] O. Vasicek, "An equilibrium characterization of the term structure," Journal of Financial Economics, vol. 5, no. 2, pp. 177-188, 1977.

[2] J. C. Cox, J. E. Ingersoll,, and S. A. Ross, "An intertemporal general equilibrium model of asset prices," Econometrica, vol. 53, no. 2, pp. 363-384, 1985.

[3] F. A. Longstaff, "A nonlinear general equilibrium model of the term structure of interest rates," Journal of Financial Economics, vol. 23, no. 2, pp. 195-224, 1989.

[4] D. H. Ahn and B. Gao, "A parametric nonlinear model of term structure dynamics," Review of Financial Studies, vol. 12, no. 4, pp. 721-762, 1999.

[5] C. F. Lo and C. H. Hui, "Valuation of financial derivatives with time-dependent parameters: lie-algebraic approach," Quantitative Finance, vol. 1, no. 1, pp. 73-78, 2001. 
[6] C. F. Lo and C. H. Hui, "Pricing multi-asset financial derivatives with time-dependent parameters-lie algebraic approach," International Journal of Mathematics and Mathematical Sciences, vol. 32, no. 7, pp. 401-410, 2002.

[7] C. F. Lo and C. H. Hui, "Lie-algebraic approach for pricing moving barrier options with time-dependent parameters," Journal of Mathematical Analysis and Applications, vol. 323, no. 2, pp. 1455-1464, 2006.

[8] J. Wei and E. Norman, "Lie algebraic solution of linear differential equations," Journal of Mathematical Physics, vol. 4, no. 4, pp. 575-581, 1963.

[9] N. H. Ibragimov and C. W. Soh, "Solution of the Cauchy problem for the Black-Scholes equation using its symmetries," in Proceedings of the International Conference on Modern Group Analysis, N. H. Ibragimov, K. R. Naqvi, and E. Straume, Eds., MARS, Nordfjordeid, Norway, 1997.

[10] R. K. Gazizov and N. H. Ibragimov, "Lie symmetry analysis of differential equations in finance," Nonlinear Dynamics, vol. 17, no. 4, pp. 387-407, 1998.

[11] P. E. Hydon, Symmetry Methods for Differential Equations: A Beginner's Guide, Cambridge University, New York, NY, USA, 2000.

[12] G. W. Bluman, A. F. Cheviakov, and S. C. Anco, Applications of Symmetry Methods to Partial Differential Equations, Springer, New York, NY, USA, 2010.

[13] J. Goard, "New solutions to the bond-pricing equation via Lie's classical method," Mathematical and Computer Modelling, vol. 32, no. 3-4, pp. 299-313, 2000.

[14] C. A. Pooe, F. M. Mahomed, and C. W. Soh, "Fundamental solutions for zero-coupon bond pricing models," Nonlinear Dynamics, vol. 36, no. 1, pp. 69-76, 2004.

[15] J. Goard, P. Broadbridge, and G. Raina, "Tractable forms of the bond pricing equation," Mathematical and Computer Modelling, vol. 40, no. 1-2, pp. 151-172, 2004.

[16] P. G. L. Leach, J. G. O’Hara, and W. Sinkala, "Symmetry-based solution of a model for a combination of a risky investment and a riskless investment," Journal of Mathematical Analysis and Applications, vol. 334, no. 1, pp. 368-381, 2007.

[17] W. Sinkala, P. G. L. Leach, and J. G. O’Hara, “Zero-coupon bond prices in the Vasicek and CIR models: their computation as group-invariant solutions," Mathematical Methods in the Applied Sciences, vol. 31, no. 6, pp. 665-678, 2008.

[18] I. Hernández, C. Mateos, J. Núñez, and Á. F. Tenorio, "Lie theory: applications to problems in mathematical finance and economics," Applied Mathematics and Computation, vol. 208, no. 2, pp. 446-452, 2009.

[19] A. Perelomov, Generalized Coherent States and Their Applications, Springer, New York, NY, USA, 1986.

[20] Y. Ait-Sahalia, "Testing continuous-time models of the spot interest rate," Review of Financial Studies, vol. 9, no. 2, pp. 385426, 1996.

[21] K. C. Chan, G. A. Karolyi, F. Longstaff, and A. B. Sanders, "An empirical comparison of alternative models of the short-term interest rate," Journal of Finance, vol. 47, pp. 1209-1227, 1992.

[22] J. Y. Campbell, A. W. Lo, and A. C. MacKinlay, The Econometrics of Financial Markets, Princeton University, Princeton, NJ, USA, 1996.

[23] I. S. Gradshteyn and I. M. Ryzhik, Table of Integrals, Series, and Products, Academic Press, Boston, Mass, USA, 5th edition, 1994.

[24] N. N. Lebedev, Special Functions and Their Applications, Dover Publications, New York, NY, USA, 1972.
[25] P. Wilmott, S. Howison, and J. Dewynne, The Mathematics of Financial Derivatives: A Student Introduction, Cambridge University, New York, NY, USA, 1989.

[26] W. H. Louisell, Quantum Statistical Properties of Radiation, John Wiley \& Sons, New York, NY, USA, 1990. 


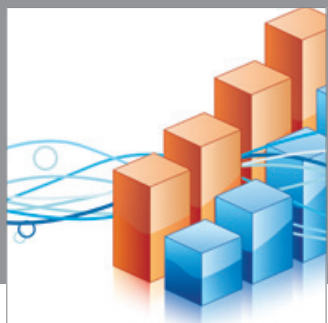

Advances in

Operations Research

mansans

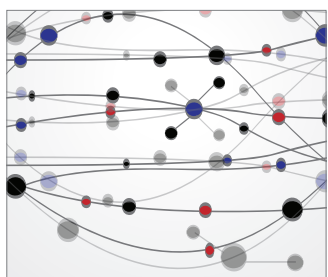

The Scientific World Journal
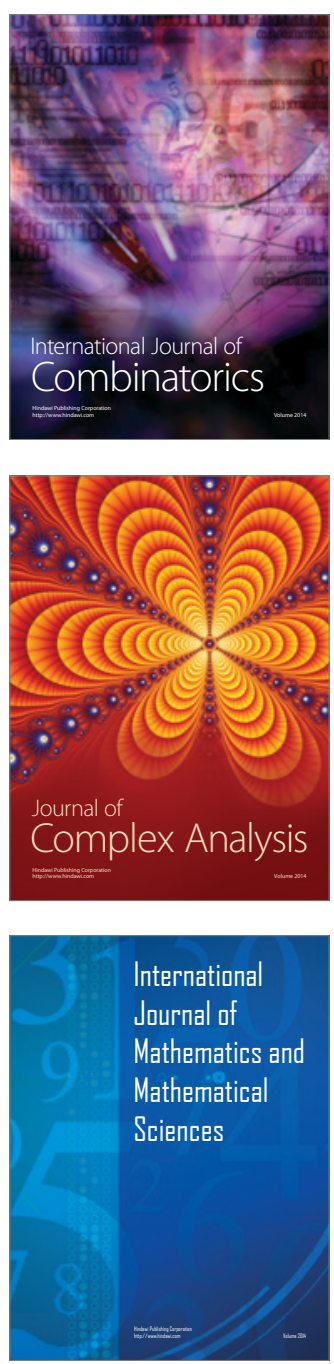
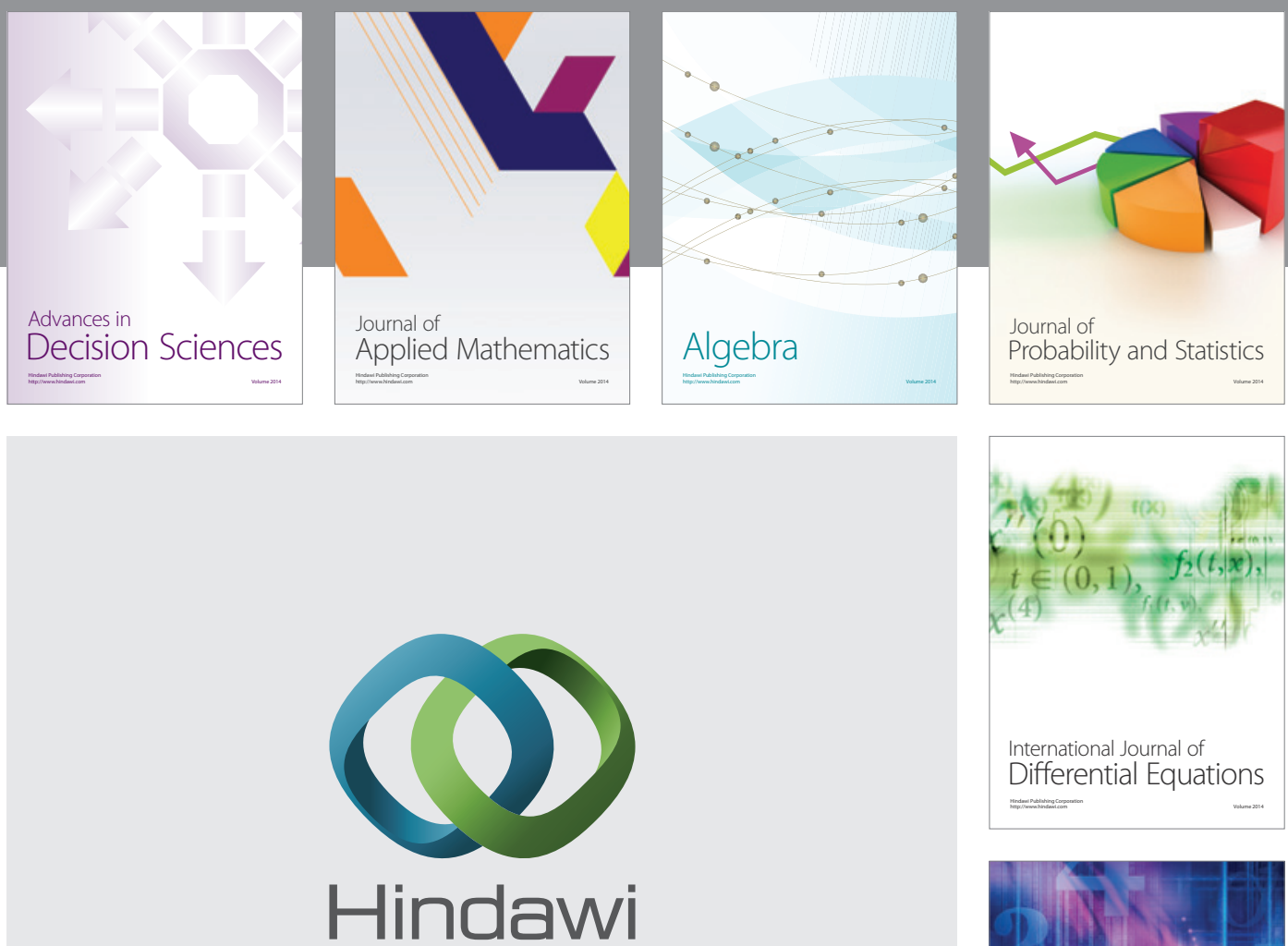

Submit your manuscripts at http://www.hindawi.com
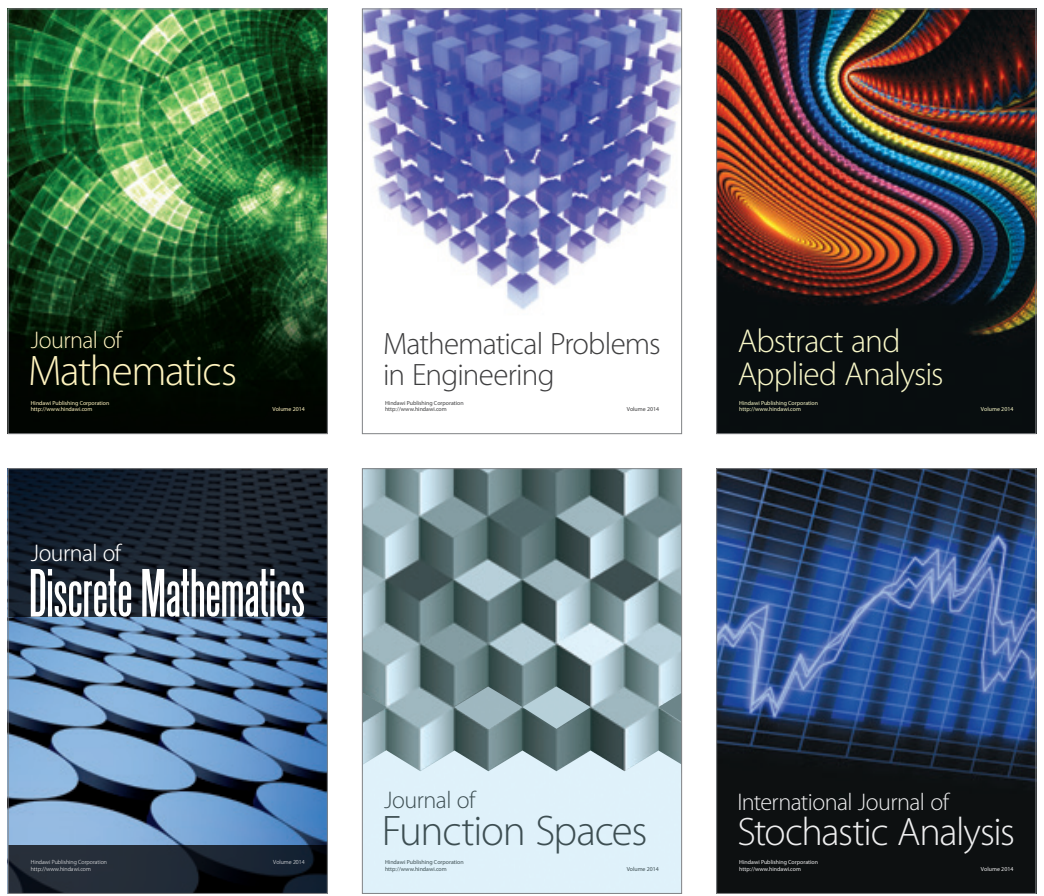

Journal of

Function Spaces

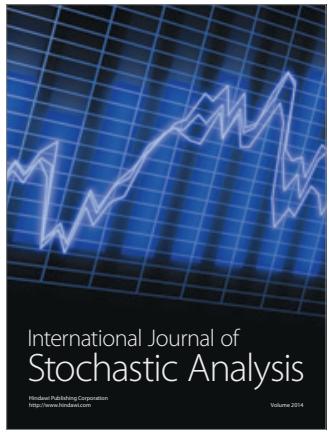

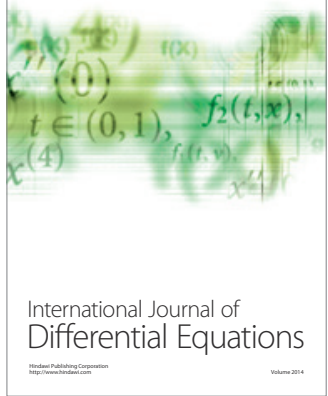
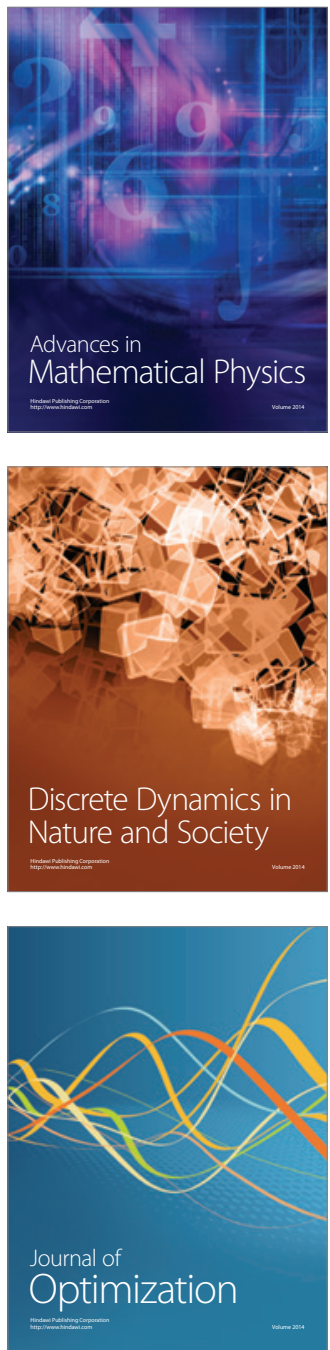